\title{
NIVEL COGNITIVO SOBRE FACTORES DE RIESGOS OCUPACIONALES Y EL GRADO DE EXPOSICIÓN EN ESTUDIANTES DEL IX Y X CICLO DE LA FACULTAD DE ENFERMERÍA DE LA UNIVERSIDAD NACIONAL SAN LUIS GONZAGA, 2019
}

Cognitive level on occupational risk factors and the degree of exposure in students of the IX and $X$ cycle of the nursing Faculty of the National University San Luis Gonzaga, 2019

\section{Alfredo Deyvi Injante Espinot,a}

${ }^{1}$ Facultad de Enfermería. Universidad Nacional San Luis Gonzaga. Ica, Perú.

aLicenciado en Enfermería

\section{RESUMEN}

El estudio tuvo por Objetivo: Identificar el nivel cognitivo sobre factores de riesgos ocupacionales y el grado de exposición en los estudiantes del IX y X ciclo de la Facultad de Enfermería de la Universidad Nacional San Luis Gonzaga, 2019. Material y Métodos: Estudio cuantitativo, descriptivo, de corte transversal. La muestra estuvo constituida por 103 estudiantes de Enfermería, la técnica utilizada fue la encuesta y el instrumento fue el cuestionario. Resultados: El conocimiento de los estudiantes sobre riesgos ocupacionales; el 53,40\% tiene conocimiento regular, el $42,72 \%$ de estudiantes tiene conocimiento bueno y solo el 3,88\% de estudiantes tiene conocimiento deficiente. El $55,34 \%$ de estudiantes tiene un grado de exposición a riesgos ocupacionales moderado, el $31,07 \%$ de estudiantes tiene un grado de exposición alto y el 14,56\% de estudiantes tiene un grado de exposición bajo. Conclusiones: Con un 95\% de confianza los datos dan evidencia que el nivel cognitivo es regular y el grado de exposición a riesgos ocupacionales es moderado en los estudiantes del IX y X ciclo de la Facultad de Enfermería de la Universidad Nacional San Luis Gonzaga.

Palabras Clave: Nivel cognitivo, grado de exposición, riesgos ocupacionales, estudiantes.

\section{SUMMARY}

The study aimed to Objective: Identify the cognitive level on occupational risk factors and the degree of exposure in the students of the IX and X cycle of the Faculty of Nursing of the National University San Luis Gonzaga, 2019. Material and Methods: Quantitative study, descriptive, crosssectional. The sample consisted of 103 nursing students, the technique used was the survey and the instrument was the questionnaire. Results: Students' knowledge about occupational risks; $53.40 \%$ have regular knowledge, $42.72 \%$ of students have good knowledge and only $3.88 \%$ of students have poor knowledge. 55.34\% of students have a moderate degree of exposure to occupational hazards, $31.07 \%$ of students have a high degree of exposure and $14.56 \%$ of students have a low degree of exposure. Conclusions: With 95\% confidence, the data give evidence that the cognitive level is regular and the degree of exposure to occupational risks is moderate in the students of the IX and X cycle of the Faculty of Nursing of the National University San Luis Gonzaga.

Key words: Cognitive level, degree of exposure, occupational risks, students.

\section{INTRODUCCIÓN.}

El estudio identificó los conocimientos sobre el riesgo ocupacional y la exposición del estudiante en el aspecto ocupacional ya que es la correlación de la posibilidad que la persona sufra un determinado daño ocasionado por el trabajo exponiéndose a componentes químicos y biológicos peligrosos (1). Los profesionales y estudiantes de enfermería, al momento de realizar sus labores en una institución de salud, se exponen a muchos riesgos ocupacionales, procedente del manejo de los fluidos $y / 0$ secreciones biológicas, 
componentes químicos, componentes ergonómicos, y situaciones psicosociales que podría afectar no solo la vitalidad, vigor y salud sino también el desempeño profesional (2). Igualmente, durante la vida profesional de un interno de enfermería, es común la aparición de acontecimientos de lumbalgias; por lo menos, un acontecimiento de lumbalgia. Así mismo el profesional del sector salud sufre un aproximando de 2 millones de hincadas con agujas al año, que eventualmente terminan en situaciones de infección por hepatitis B y VIH (2). En este contexto según revisión bibliográfica se pudo evidenciar que "de los 35 millones de trabajadores que laboran en el sector salud en todo el mundo, cada año aproximadamente 3 millones de ellos se exponen a una exposición percutánea de los patógenos que se transmiten por los fluidos y la sangre; se cree que por esta razón existen 16000 casos de hepatitis C; 66000 de hepatitis B y de 200 a 5000 infecciones por el (VIH). Se observa que más del $90 \%$ de dichas infecciones son ocasionadas en los países que aún se encuentran en desarrollo y la mayoría es prevenible" (3). El Ministerio de Salud reporto que en primer lugar el porcentaje de todos los trabajadores que se encuentran expuestos a los factores de riesgos ocupacionales, es la exposición que se da hacia los factores de riesgo físico en un $27 \%$ de los casos, en segundo lugar la exposición a factores causales de accidentes en un $23,1 \%$, en tercer lugar exposición ante los factores de riesgo ergonómico en un $17,6 \%$, encuarto lugar se encuentra la exposición hacia los factores de riesgo biológico con un $14,2 \%$, en quinto lugar la exposición hacia los factores de riesgo químico con un $11,6 \%$ y en último lugar los expuestos ante los factores de riesgo psicosocial en un 6,5\%. Con relación a los riesgos biológicos, se encontraron 6,099 trabajadores de la salud registrados, donde solo el $77,0 \%$ corresponden a los servicios de salud (4). En este sentido, haciendo una revisión bibliográfica al respecto, investigaciones realizadas: en Colombia año 2017 se obtuvo que el $58 \%$ del personal tienen conocimientos regulares acerca de los riesgos laborales, el $12 \%$ tienen conocimientos deficientes y el $20 \%$ tienen conocimientos buenos (5). En España, año 2017 el estudio, registró el 71\% del personal presentan conocimientos regulares sobre prevención de riesgos laborales y el $29 \%$ tienen conocimientos deficientes (6). En Huancavelica investigación realizada en el 2017 predomino que el $50 \%$ de los profesionales de enfermería presentaron riesgo laboral medio (7). La tesis ejecutada año 2015 predomino que el $70 \%$ de los profesionales de enfermería presentaban un nivel de conocimiento regular y el $100 \%$ se encontraba expuesta a riesgos ocupacional alto (8). En Lima año 2017 la investigación reportó que del $100 \%$ de enfermeras, el $56 \%$ expresan que está presente el riesgo laboral y $44 \%$ ausente (9). El autor Ávila $\mathrm{R}$ en el 2017 realizo un estudio titulado Factores de riesgo laboral en enfermería del Hospital María Auxiliadora donde concluyo que los factores de riesgo laboral se encontraban presentes (10).

El estudio se justifica porque hay una alta incidencia de estudiantes expuestos a factores de riesgo ocupacionales cuando realizan sus prácticas pre profesionales y también es relevante por considerarse la prevención uno de los pilares principales dentro de toda institución que cuenta con capital humano. Es importante el estudio porque los datos que se obtienen en la investigación brindaran información actualizada pudiendo utilizarla como base de datos y evidencias sobre los riesgos laborales y ayudar de esta manera con información actual acerca de los riesgos a los que están expuestos los estudiantes de enfermería. Señalándose como objetivo determinar el nivel cognitivo sobre factores de riesgos ocupacionales y el grado de exposición en los estudiantes del IX y X ciclo de la Facultad de Enfermería de la Universidad Nacional San Luis Gonzaga, 2019. 


\section{MATERIAL Y MÉTODOS.}

El estudio es de tipo descriptivo de corte transversal no experimental. La población se encuentra conformada por los todos los estudiantes del IX y $X$ ciclo de la facultad enfermería de la universidad nacional San Luis Gonzaga de Ica, el tamaño de la muestra por conveniencia se constituye por 103 estudiantes de enfermería. La técnica utilizada para la recolección de datos fue la encuesta y el instrumento utilizado fue el cuestionario aplicando la prueba piloto obteniendo una confiabilidad Alfa de Cronbach de 0,842 para el cuestionario de Nivel cognitivo, y 0,831 para el cuestionario de Grado de Exposición. La Variable Nivel Cognitivo contiene 4 dimensiones con 20 ítems, y la variable Grado de Exposición contiene 5 dimensiones con 25 ítems. La variable Nivel cognitivo se realizó utilizando un formulario tipo escala vigesimal con una medición de nivel: Bueno, Regular y Deficiente, la variable Grado de Exposición se realizó utilizando la escala de Likert con los índices (Siempre, A veces, Nunca) con un nivel de medición de Bajo, Moderado y Alto. Para el procesamiento de datos se utilizó la estadística descriptiva y el uso del software SPSS versión 22. Para la contratación de hipótesis se utilizó la prueba estadística Chi cuadrado $\left(\mathrm{X}_{2}\right)$ con un nivel de confiabilidad del $95 \%$, obteniendo un valor de 6,478 aceptando la $\mathrm{H}_{1}$.

\section{RESULTADOS.}

En relación a la edad de los estudiantes de la facultad de enfermería de la universidad Nacional San Luis Gonzaga predomino la edad de 21 años con 42,72\% (41 alumnos), en relación al sexo de los estudiantes de enfermería predomino el género Femenino con 92,23\% (95 alumnas), respecto a la rotación de prácticas de los estudiantes predomino la rotación Hospitalaria con 69,90\% (72 alumnos). En la Tabla 1 se analizó el Nivel Cognitivo sobre Riesgos Ocupacionales, obteniendo que el 53,40\% de estudiantes presentan conocimiento regular, el $42,72 \%$ tienen conocimiento bueno y solo el 3,88\% tienen conocimiento deficiente. En el Grafico 1 se analizó el Nivel Cognitivo sobre la Dimensión Riesgos Biológicos obteniendo como resultados que el $51,46 \%$ de estudiantes presentan conocimiento bueno, el $47,57 \%$ tienen conocimiento regular y solo el $0,97 \%$ tienen conocimiento deficiente. Se analizó el Nivel Cognitivo sobre la Dimensión Riesgos Químicos obteniendo como resultados que el $63,11 \%$ de estudiantes presentan conocimiento regular, el $33,98 \%$ tienen conocimiento bueno y solo el $2,91 \%$ tienen conocimiento deficiente. Seguido del Nivel Cognitivo sobre la Dimensión Riesgos Ergonómicos obteniendo como resultados que el $40,78 \%$ de estudiantes presentan conocimiento bueno, el $57,28 \%$ tienen conocimiento regular y solo el $1,94 \%$ tienen conocimiento deficiente. Por último, se analizó el Nivel Cognitivo sobre la Dimensión Riesgos Psicosociales obteniendo como resultados que el $59,22 \%$ de estudiantes presentan conocimiento regular, el 39,80\% tienen conocimiento bueno y solo el $0,98 \%$ tienen conocimiento deficiente. En la Tabla 2 se analizó el Grado de Exposición a Riesgos Ocupacionales obteniendo como resultados que el $55,34 \%$ de estudiantes presentan un grado de exposición a riesgos ocupacionales moderado, el 31,07\% tienen un grado de exposición alto y el $14,56 \%$ tienen un grado de exposición bajo. En el Grafico 2 se analizó el Grado de Exposición a Riesgos Físicos obteniendo como resultados que el $69,90 \%$ de estudiantes presentan un grado de exposición a riesgos físicos moderado, el $25,24 \%$ tienen un grado de exposición alto y el $4,85 \%$ tienen un grado de exposición bajo. Se analizó el Grado de Exposición a Riesgos Ergonómicos obteniendo como resultados que el $78,93 \%$ de estudiantes presentan un grado de exposición a riesgos ergonómicos moderado, el $27,18 \%$ tienen un grado de exposición alto y el 3,88\% tienen un grado de exposición bajo. Se analizó el Grado de Exposición a Riesgos Psicosociales obteniendo como resultados que el $76,70 \%$ de estudiantes presentan un grado de 
exposición a riesgos psicosociales moderado, el $16,50 \%$ tienen un grado de exposición alto y el 6,80\% tienen un grado de exposición bajo. Seguido del Grado de Exposición a Riesgos Biológicos obteniendo como resultado que el $78,64 \%$ de estudiantes presentan un grado de exposición a riesgos biológicos moderado, el $11,65 \%$ tienen un grado de exposición alto y el 9,71\% tienen un grado de exposición bajo. Por último, se analizó el Grado de Exposición a Riesgos Químicos obteniendo como resultados que el $83,50 \%$ de estudiantes presentan un grado de exposición a riesgos químicos moderado, el $11,65 \%$ tienen un grado de exposición alto y el $8,45 \%$ tienen un grado de exposición bajo.

Tabla 1. Nivel Cognitivo sobre Riesgos Ocupacionales

\begin{tabular}{ccc}
\hline Conocimiento & $\mathbf{N}^{\circ}$ & $\%$ \\
\hline C. Bueno & 44 & $42,72 \%$ \\
\hline C. Regular & 55 & $53,40 \%$ \\
\hline C. Deficiente & 04 & $3,88 \%$ \\
\hline Total & 103 & $100,00 \%$ \\
\hline
\end{tabular}

Fuente: Base de datos

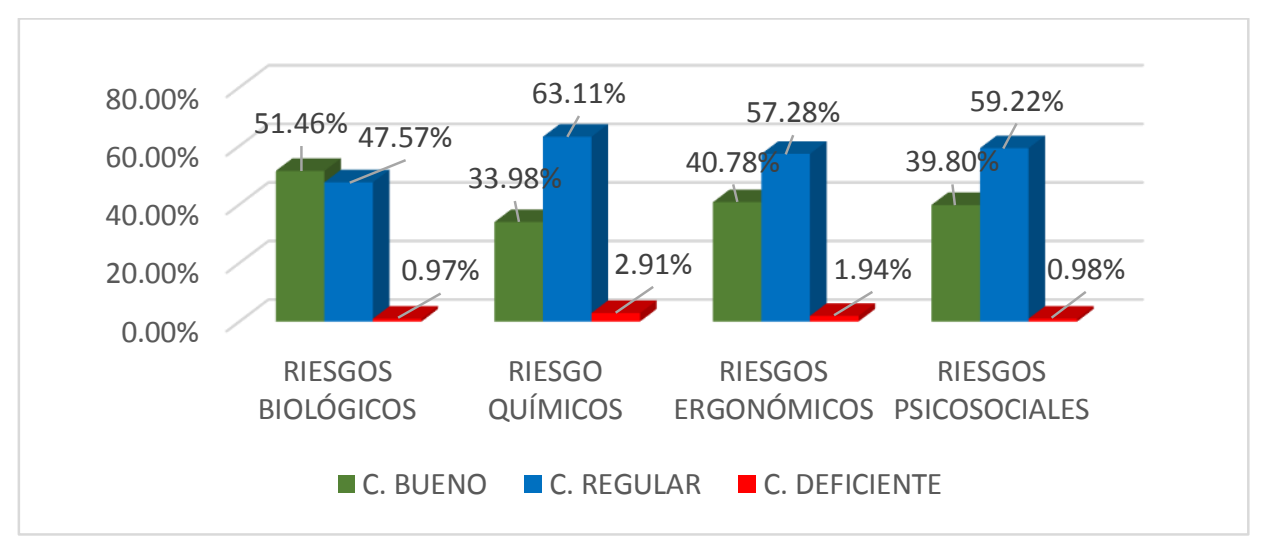

Gráfico 1. Nivel Cognitivo sobre las dimensiones de riesgos biológicos, Químicos, Ergonómicos, Psicosociales

Tabla 2. Grado de Exposición a Riesgos Ocupacionales.

\begin{tabular}{ccc}
\hline Grado de Exposición & $\mathbf{N}^{\circ}$ & $\%$ \\
\hline Grado Alto & 32 & $31,07 \%$ \\
\hline Grado Moderado & 57 & $55,34 \%$ \\
\hline Grado Bajo & 15 & $14,56 \%$ \\
\hline Total & 103 & $100,00 \%$ \\
\hline
\end{tabular}

Fuente: Base de datos

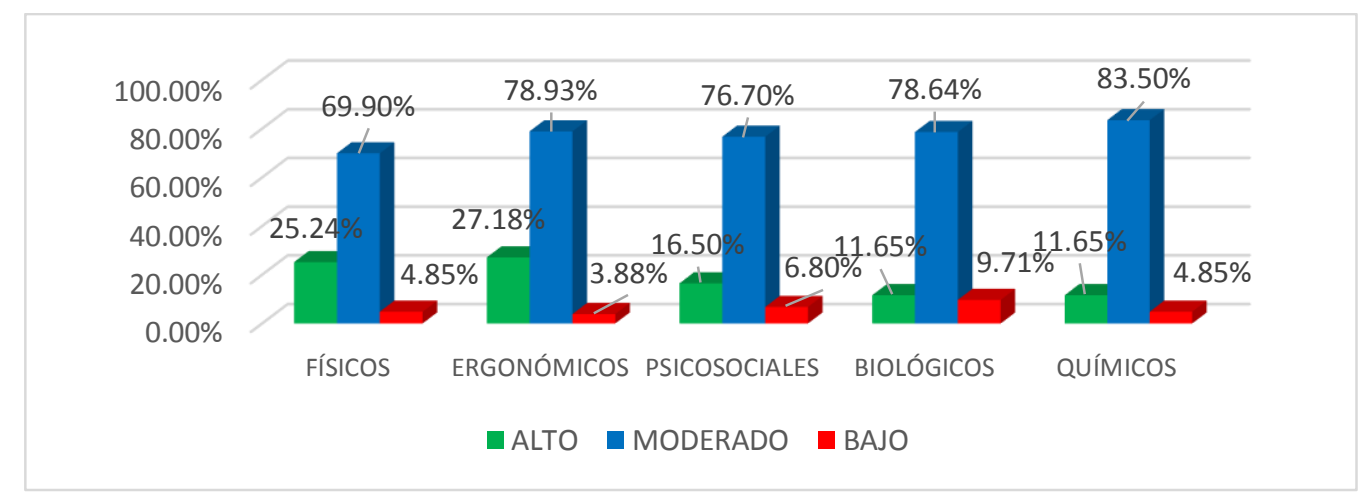

Gráfico 2. Grado de exposición sobre las dimensiones a riesgos: físicos, ergonómicos, psicosociales, biológicos y químicos. 


\section{DISCUSIÓN.}

En el estudio se evidencio que el conocimiento sobre riesgos ocupacionales en estudiantes; el $53,40 \%$ presentan conocimiento regular, el $42,72 \%$ tienen conocimiento bueno y solo el $3,88 \%$ tienen conocimiento deficiente; similar al estudio de Solís L, Flores L y Zambrano B, en su estudio: Conocimientos y el tiempo de exposición a riesgos laborales, el nivel de conocimientos es bajo (5). Ortiz D. refiere que el $70 \%$ de licenciadas en enfermería muestran conocimiento regular, el $25 \%$ con un nivel alto y solamente un $5 \%$ con un nivel bajo (8).

En el riesgo químico; el $63,11 \%$ de estudiantes tienen conocimiento regular, el $33,98 \%$ tienen conocimiento bueno y solo el $2,91 \%$ tienen conocimiento deficiente. Las enfermeras en sus actividades diarias están en contacto con diversas sustancias químicas, si no aplican las medidas preventivas puede ser perjudicial para la salud conllevando a daños en diversos órganos provocando mutación celular y cáncer, al desarrollo de enfermedades respiratorias por no utilizar la mascarilla, malformaciones congénitas, y dermatitis por el lavado de manos frecuentemente. Entre las sustancias que se utilizan en los hospitales, mayor atención el glutaraldehído, antiséptico como la clorhexidina y medicamentos tóxicos.

En el riesgo ergonómico; el 40,78\% de estudiantes tienen conocimiento bueno, el $57,28 \%$ tienen conocimiento regular y solo el $1,94 \%$ tienen conocimiento deficiente. Los problemas ergonómicos están relacionados al uso de instrumentos médicos como silla de ruedas, se ha podido observar que el personal de enfermería emplea posturas inadecuadas al momento de realizar alguna actividad o procedimiento, por esta razón es que el personal de enfermería se ve expuesto a diversas lesiones como son: lumbalgias, hernias, alteraciones circulatorias, debilidad muscular.
En el riesgo psicosocial; el 59,22\% de estudiantes tienen conocimiento regular, el $39,80 \%$ tienen conocimiento bueno y solo el $0,98 \%$ tienen conocimiento deficiente.

Según la exposición a riesgos ocupacionales, el $55,34 \%$ de estudiantes tienen un grado de exposición a riesgos ocupacionales moderado, el 31,07\% tienen un grado de exposición alto y el $14,56 \%$ tienen un grado de exposición bajo, el cual coincide con el estudio de Jurado $\mathrm{K}$. Exposición a riesgos laborales, del 100\%, $56 \%$ expresan que está presente el riesgo laboral y $44 \%$ ausente (9). Escobar D. Riesgos laborales de los profesionales. Solo el $50 \%$ presentaron un riesgo laboral medio siguiendo de un de 33,3\% como riesgo bajo (7). Ávila R. Factores de riesgo laboral, el $54 \%$ de los factores de riesgos laboral estaban presentes y solo el $46 \%$ no está presente el riesgo (10).

Según la exposición a riesgos ocupacionales, el $55,34 \%$ de estudiantes tienen un grado del $78,93 \%$ de estudiantes tienen un grado de exposición a riesgos ergonómicos moderado, el $27,18 \%$ de estudiantes tienen un grado de exposición alto y el $3,88 \%$ de estudiantes tienen un grado de exposición bajo, el cual coincide con el estudio de Escobar D. Respecto a riesgo ergonómico se encontró que el 63,3\% presentaron riesgo ergonómico medio, $20,0 \%$ riesgo bajo y $16,7 \%$ riesgo alto (7).

El $78,93 \%$ de estudiantes tienen un grado de exposición a riesgos ergonómicos moderado, el $27,18 \%$ de estudiantes tienen un grado de exposición alto y el 3,88\% de estudiantes tienen un grado de exposición bajo, el cual coincide con el estudio de Escobar D. Respecto a riesgo ergonómico se encontró que el $63,3 \%$ presentaron riesgo ergonómico medio, $20,0 \%$ riesgo bajo y $16,7 \%$ riesgo alto (7).

El $76,70 \%$ de estudiantes tienen un grado de exposición a riesgos psicosociales moderado, el $16,50 \%$ de estudiantes tienen un grado de exposición alto y el $6,80 \%$ de 
estudiantes tienen un grado de exposición bajo, el cual coincide con el estudio de Escobar D. encontró en los Riesgo psicosocial se encontró el 50,0\% presentan riesgo psicosocial medio (7).

El 78,64\% de estudiantes tienen un grado de exposición a riesgos biológicos moderado, el $11,65 \%$ de estudiantes tienen un grado de exposición alto y el $9,71 \%$ de estudiantes tienen un grado de exposición bajo, el cual coincide con el estudio de Escobar D. donde se encontró que el $76,7 \%$ presentaron riesgo biológico medio (7).

El $83,50 \%$ de estudiantes tienen un grado de exposición a riesgos químicos moderado, el $11,65 \%$ de estudiantes tienen un grado de exposición alto y el $8,45 \%$ de estudiantes tienen un grado de exposición bajo, el cual no coincide con el estudio de Escobar D. donde el riesgo químico que se encontró que el $76,7 \%$ presentan riesgo bajo (7).

\section{CONCLUSIONES.}

1. El nivel cognitivo en una tasa global de los estudiantes sobre riesgos ocupacionales es regular en la mayoría equivalente a un $53,40 \%$; el $55,34 \%$ de estudiantes tienen un grado de exposición a riesgos ocupacionales moderado, siendo el más alto índice.

2. El conocimiento de los estudiantes sobre riesgo biológico; el $51,46 \%$ tienen conocimiento bueno, el $47,57 \%$ de estudiantes tienen conocimiento regular y solo el $0,97 \%$ de estudiantes tienen conocimiento deficiente.

3. El conocimiento de los estudiantes sobre riesgo químico; el $63,11 \%$ tienen conocimiento regular, el $33,98 \%$ de estudiantes tienen conocimiento bueno y solo el $2,91 \%$ de estudiantes tienen conocimiento deficiente.

4. El conocimiento de los estudiantes sobre riesgo ergonómico; el 40,78\% tienen conocimiento bueno, el $57,28 \%$ de estudiantes tienen conocimiento regular y solo el $1,94 \%$ de estudiantes tienen conocimiento deficiente.
5. El conocimiento de los estudiantes sobre riesgo psicosocial; el $59,22 \%$ de estudiantes tienen conocimiento regular, el 39,80\% tienen conocimiento bueno y solo el $0,98 \%$ de estudiantes tienen conocimiento deficiente.

6. El $55,34 \%$ de estudiantes tienen un grado de exposición a riesgos ocupacionales moderado, el $31,07 \%$ de estudiantes tienen un grado de exposición alto y el $14,56 \%$ de estudiantes tienen un grado de exposición bajo.

\section{Correspondencia.}

Lic. en Enf. Alfredo Deyvi Injante Espino

Correo electrónico:

alfredoinjante@gmail.com

\section{REFERENCIAS BIBLIOGRAFICAS.}

1. EsSalud. Enfermedades ocupacionales e Higiene ocupacional. Boletín EsSalud. 2016; (5).

2. De Pablo C. Riesgos laborales del personal de enfermería en quirófano. 2a ed. España: Formación Alcalá; 2017.

3. Mansilla F. Manual de riesgos psicosociales en el trabajo: teoría y práctica. Madrid: Madrid Salud; 2016.

4. Ministerio de Salud. "Manual de Salud Ocupacional” (internet). Perú. 2016. (Citado el 10 de Noviembre del 2019). Disponible en: http://www.digesa.minsa.gob.pe/publica ciones/descargas/manual deso.PDF

5. Solís L, Flores $\mathbf{L}$ y Zambrano B. Conocimientos y el tiempo de exposición a riesgos laborales del personal adscrito a quirófano en un hospital para personas sin seguridad social. Rev Colombiana de Salud Ocupacional. 2017; 7(1): 16-21. 
6. Arce S. La Prevención de Riesgos Laborales y la accidentalidad laboral en la prensa española: representación y cobertura a partir de la Ley 31/1995 de Prevención de Riesgos Laborales. Tesis doctoral. Burgos, España. Universidad de Burgos. 2017.

7. Escobar D. Riesgos laboral de los profesionales de Enfermería en el Servicio de Cirugía y Medicina del Hospital Regional Zacarías Correa Valdivia de Huancavelica. Tesis Pregrado. Huancavelica, Perú. Escuela Profesional de Enfermería, Universidad Nacional de Huancavelica; 2017.

8. Ortiz D. Nivel de conocimiento y el grado de exposición al riesgo ocupacional del personal de enfermería del centro de salud Nuevo Chimbote, 2015. Tesis de Especialidad. Trujillo, Perú. Facultad de Enfermería, Segunda Especialidad, Universidad Nacional de Trujillo; 2015.
9. Jurado K. Exposición a riesgos laborales del personal de enfermería en Sala de Operaciones del Hospital III Emergencias Grau. Tesis de Especialidad. Lima, Perú. Facultad de Medicina, Programa de Segunda Especialización en Enfermería, Universidad Nacional Mayor de San Marcos; 2017.

10. Ávila R. Factores de riesgo laboral en profesionales de enfermería servicio de emergencia Hospital María Auxiliadora. Tesis Pre-grado. Lima, Perú. Facultad de Enfermería, Universidad Privada San Juan Bautista; 2017.

Recibido: 18/07/2020

Aprobado para publicación: 19/10/2020 